\title{
Empirical analysis suggests continuous and homogeneous circulation of Newcastle disease virus in a wide range of wild bird species in Africa
}

\author{
J. CAPPELLE ${ }^{1,2 *}$, A. CARON ${ }^{1,3,4}$, R. SERVAN De ALMEIDA ${ }^{5,6}$, P. GIL $^{5,6}$, \\ M. PEDRONO ${ }^{1}$, J. MUNDAVA $^{7}$, B. FOFANA ${ }^{8}$, G. BALANÇA $^{1}$, M. DAKOUO ${ }^{9}$, \\ A. B. OULD EL MAMY ${ }^{10}$, C. ABOLNIK ${ }^{11}$, O. F. MAMINIAINA ${ }^{12}$, G. S. CUMMING ${ }^{13}$, \\ M.-N. De VISSCHER ${ }^{1}$, E. ALBINA ${ }^{6,14}$, V. CHEVALIER ${ }^{1}$ AND N. GAIDET ${ }^{1}$ \\ ${ }^{1}$ CIRAD-ES, UR AGIRs, F34398 Montpellier, France; ${ }^{2}$ Institut Pasteur du Cambodge, Epidemiology and Public \\ Health Unit, Phnom Penh, Cambodia; ${ }^{3}$ CIRAD-RP-PCP, UR AGIRs, Harare, Zimbabwe; ${ }^{4}$ Mammal Research \\ Institute, University of Pretoria, Pretoria, South Africa; ${ }^{5}$ CIRAD-BIOS, UMR CMAEE, F34398 Montpellier, \\ France; ${ }^{6}$ INRA, UMR1309 CMAEE, F34398 Montpellier, France; ${ }^{7}$ National University of Science and \\ Technology, Bulawayo, Zimbabwe; ${ }^{8}$ Direction Nationale des Eaux et Forêts, Bamako, Mali; ${ }^{9}$ Laboratoire \\ Central Vétérinaire, Bamako, Mali; ${ }^{10}$ Centre National d'Elevage et de Recherche Vétérinaires (CNERV), \\ Nouakchott, Mauritania; ${ }^{11}$ Poultry Section, Department of Production Animal Studies, Faculty of Veterinary \\ Science, University of Pretoria, Onderstepoort, South Africa; ${ }^{12}$ FOFIFA-DRZV, Antananarivo, Madagascar; \\ ${ }^{13}$ Percy FitzPatrick Institute, University of Cape Town, Rondebosch, Cape Town 7701, South Africa; \\ ${ }^{14}$ CIRAD-BIOS, UMR CMAEE, F-97117 Petit-Bourg, Guadeloupe, France
}

Received 12 April 2014; Final revision 18 June 2014; Accepted 28 June 2014;

first published online 4 August 2014

\section{SUMMARY}

Newcastle disease (ND) is one of the most important poultry diseases worldwide and can lead to annual losses of up to $80 \%$ of backyard chickens in Africa. All bird species are considered susceptible to ND virus (NDV) infection but little is known about the role that wild birds play in the epidemiology of the virus. We present a long-term monitoring of 9000 wild birds in four African countries. Overall, $3 \cdot 06 \%$ of the birds were PCR-positive for NDV infection, with prevalence ranging from $0 \%$ to $10 \%$ depending on the season, the site and the species considered. Our study shows that ND is circulating continuously and homogeneously in a large range of wild bird species. Several genotypes of NDV circulate concurrently in different species and are phylogenetically closely related to strains circulating in local domestic poultry, suggesting that wild birds may play several roles in the epidemiology of different NDV strains in Africa. We recommend that any strategic plan aiming at controlling ND in Africa should take into account the potential role of the local wild bird community in the transmission of the disease.

Key words: APMV-1, ecology, epidemiology, infection, maintenance, Madagascar, Mali Mauritania, spread, transmission, Zimbabwe.

\section{INTRODUCTION}

Newcastle disease is one of the most important poultry diseases worldwide. It is a particular threat to food safety in most developing African and Asian countries where chickens are an important source of protein and income [1,2]. This disease is caused by the avian paramyxovirus type 1 (APMV-1), also

\footnotetext{
* Author for correspondence: Dr J. Cappelle, CIRAD, AGIRs Research Unit (Animal and Integrated Risk Management, UPR22), Intitut Pasteur du Cambodge, BP 983, Phnom Penh, Cambodia. (Email: julien.cappelle@cirad.fr)
} 
named Newcastle disease virus (NDV), classified in two main classes of several genotypes each. Class I is divided into genotypes $1-9$ while class II is divided into genotypes I-XIV [3, 4]. Strains of NDV are also characterized according to their pathogenicity for chickens as either low pathogenic NDV (LPNDV) or virulent NDV (vNDV) [3]. The virulent form is listed in the World Organization for Animal Health (OIE) Terrestrial Animal Health Code and detection thereof must be notified to the OIE. Outbreaks of vNDV are associated with high morbidity and mortality rates of up to $100 \%$ in gallinaceous poultry species and can lead to annual losses of up to $80 \%$ of backyard chickens raised in developing countries where the disease is endemic [2]. As the clinical and epidemiological features of vNDV are similar to highly pathogenic avian influenza (HPAI) viruses, both diseases are difficult to differentiate without laboratory confirmation.

Panzootics of vNDV have occurred during the twentieth century after the first in 1926 [2]. Vaccinated domestic poultry are considered to be the main reservoir of vNDV, as the vaccines prevent mortality and production losses but permit subclinical infections with shedding, thereby maintaining vNDV in poultry populations [5-8]. Although NDV epidemiology has received some scientific attention recently, thereby enhancing our understanding of the dynamics of the viruses in poultry populations, grey areas persist. This lack in knowledge concerns the role of wild hosts in NDV introduction, maintenance and spread and specifically the epidemiology of NDV in rural production systems of developing countries, particularly in Africa [1].

The role of wild bird species in the epidemiology of $\mathrm{NDV}$, and in particular of vNDV, is unclear and their potential to spread or maintain these viruses remains poorly understood. Different LPNDV strains seem to commonly circulate in wild bird populations as illustrated by the phylogenetic diversity of LPNDV circulating in wild waterfowl and shorebirds in North America [9]. Regarding vNDV, some experimentally infected wild birds (e.g. double-crested cormorant, Phalacrocorax auritus) were shown to shed VNDV in the absence of mortality or clinical signs [10]. Various wild bird species have been also suspected to be the source of NDV outbreaks in poultry: doublecrested cormorants in Canada [11], feral pigeons (Columba livia) in North America and Europe [12] and the Eurasian collared dove (Streptopelia decaocto) in the USA [13]. Following the second panzootic that emerged in the late 1960s, numerous studies were conducted in the 1970s to determine whether wild birds may act as reservoir of NDV [2]. In an exhaustive review published in 1988, Kaleta \& Baldauf reported that 236 different bird species from 23 orders had been tested positive for NDV, mostly through natural infection [14]. They concluded that all bird species can be considered as susceptible to NDV infection and the role of certain groups of species in the epidemiology of NDV was highlighted: species living in close contact to the sea or fresh water tended to be quite resistant to vNDV while gregarious species were more likely to acquire NDV infection [14]. Most LPNDV belong to class I NDV and to genotypes I and II of class II $\mathrm{NDV}$, and have mainly been isolated from wild waterfowl and shorebirds which as a result are considered the main reservoirs of these strains [3]. Some LPNDV strains isolated in poultry in live bird markets were phylogenetically related to LPNDV strains isolated in wild birds, suggesting that they may occasionally be transmitted between wild and domestic populations [15]. Some of these LPNDV strains have also the potential to be the precursors of VNDV responsible for outbreaks after introduction into poultry populations as observed in Ireland and in Australia [16, 17]. However, the mechanisms and factors driving the emergence of vNDV from LPNDV circulating in wild birds, before or after introduction into poultry populations, are largely unknown [2].

Over the past decade, the emergence and spread of HPAI H5N1 has led to the monitoring of large wild bird populations across the world, albeit focused on wild waterfowl, the main reservoir of avian influenza virus (AIV). The biological material collected and the RNA extraction method are the same for AIV and for NDV, and several research groups tested their samples for both NDV and AIV. The extension of NDV studies to new geographical areas and new species confirmed the global pattern that had been described since the 1970s: a large range of species are susceptible to NDV infection, with LPNDV mostly isolated in waterfowl [18-20] and specific genotypes of vNDV persisting in certain species such as pigeons [21] and cormorants [22]. These studies also showed a high variability in the NDV infection rate of wild bird populations depending on the site, the period, the species and the methods used. Virus prevalence varied between $0.5 \%$ in Japan [23], $0 \cdot 7 \%$ in Brazil [20], $3 \cdot 4 \%$ in Australia [24], 5.18\% in Turkey [19] and $5.5 \%$ in Finland [18]. However, few studies have described the seasonal patterns of NDV infection in 
wild birds or have investigated the main ecological drivers of NDV infection in wild birds.

Here, we present a large-scale study comprising NDV long-term monitoring of $\sim 9000$ wild birds in four African countries, Madagascar, Mali, Mauritania and Zimbabwe. This monitoring programme was designed to study both AIV and NDV in African wild birds [25-27]. Thus, the study did not focus on waterfowl only and studies on NDV epidemiology were concurrently implemented in poultry [28-30]. In this paper, we focused on three questions: (i) what is the host range of NDV in wild birds in Africa and is there any variability in virus prevalence between bird species or phylum in the wild African bird community?, (ii) is there a year-round circulation of NDV in wild birds in Africa or is there any seasonal pattern?, and (iii) do NDV strains detected in domestic and wild birds indicate any exchange of virus at the wildlife/domestic interface?

\section{METHODS}

\section{Study sites}

Long-term monitoring of African wild birds was implemented in Madagascar, Mali, Mauritania and Zimbabwe through 33 sampling occasions in seven wetlands over 4 years (Fig. 1). In West Africa, samples were collected from large natural areas. The Inner Niger Delta in Mali is the largest continental wetland in West Africa supporting several millions of Afrotropical and Eurasian migratory waterbirds, and the Parc National du Banc d'Arguin in Mauritania constitutes one of the largest wintering sites for shorebirds in the world ( $\sim 2.3$ million birds). In contrast, samples were mainly collected from smaller man-made wetlands, the adjacent lakes Chivero and Manyame in Zimbabwe and Lake Alaotra in Madagascar. Except in Banc d'Arguin where few villages and domestic poultry are present, human villages are settled in these areas and most poultry are raised traditionally, free-ranging around the village. Poultry are therefore likely to share the same habitats as wild birds in the vicinity of villages, especially during the dry season when wild birds use remnant water bodies [31].

\section{Ethical standards}

During the study, birds were captured and released into the wild using conventional techniques (mist nets and baited walk-in traps) covered by the Ornithological Council's 'Guidelines for the Use of Wild Birds in
Research'. Procedures for capture, handling, and sampling were approved by the Centre de Recherches par le Baguage des Populations d'Oiseaux (CRBPO, Natural History Museum Paris-French National Reference Bird Ringing Centre). Capture permits were obtained from the relevant government authority in each country where field studies were conducted. All sampling activities were conducted in the presence of a representative from the animal health and veterinary national services and a representative from the environment national services.

\section{Sampling and diagnostic procedures}

Cloacal, tracheal, and faecal samples were collected from live-caught birds captured using mist nets and walk-in traps, from recently caught birds provided by traditional hunters or from fresh droppings collected at roosting sites. All samples collected in Madagascar, Mali and Mauritania were analysed at CIRAD Laboratory in Montpellier, France following a common procedure for collection, storage, shipment and virological testing. These samples were tested using a technique targeting class II NDV only in order to have a better sensitivity for vNDV detection. Viral RNA was extracted from samples by a high throughput automated platform Biomek FX ${ }^{\mathrm{P}}$ (Beckman, USA) using the Nucleospin RNA virus kit (Macherey Nagel, Germany). NDV was detected by a one-step real time RT-PCR (rRT-PCR) targeting the F gene with the forward primer F259 5'- ACATTGACCACTTTGCTCA-3' and the reverse primer F488rev 5'-TGCACAGCCTCATTGGTTGC-3'. The PCR fragment of $239 \mathrm{bp}$ was sequenced externally (GATC Biotech, Germany). All samples positive by RTqPCR were inoculated into the allantoic cavities of 9- to 11-day-old embryonated fowl eggs from a commercial specific-pathogen-free flock. After 1-3 passages, allantoic liquid from dead eggs was tested by NDV RT-qPCR, and NDV-positive samples were then stored at $-80^{\circ} \mathrm{C}$ and used as working stock for sequence analysis. More details on the methods are published in de Almeida et al. [4]. All samples collected in Zimbabwe were analysed at Onderstepoort Veterinary Institute (OVI) Laboratory, Onderstepoort, South Africa following a common procedure for collection, storage and shipment. RNA was extracted using either TriZOL reagent (Invitrogen, USA) or an automated MagNaPure platform (Roche, USA) and two different viral RNA procedures were used for virological testing. Samples collected between May 2007 and March 2009 


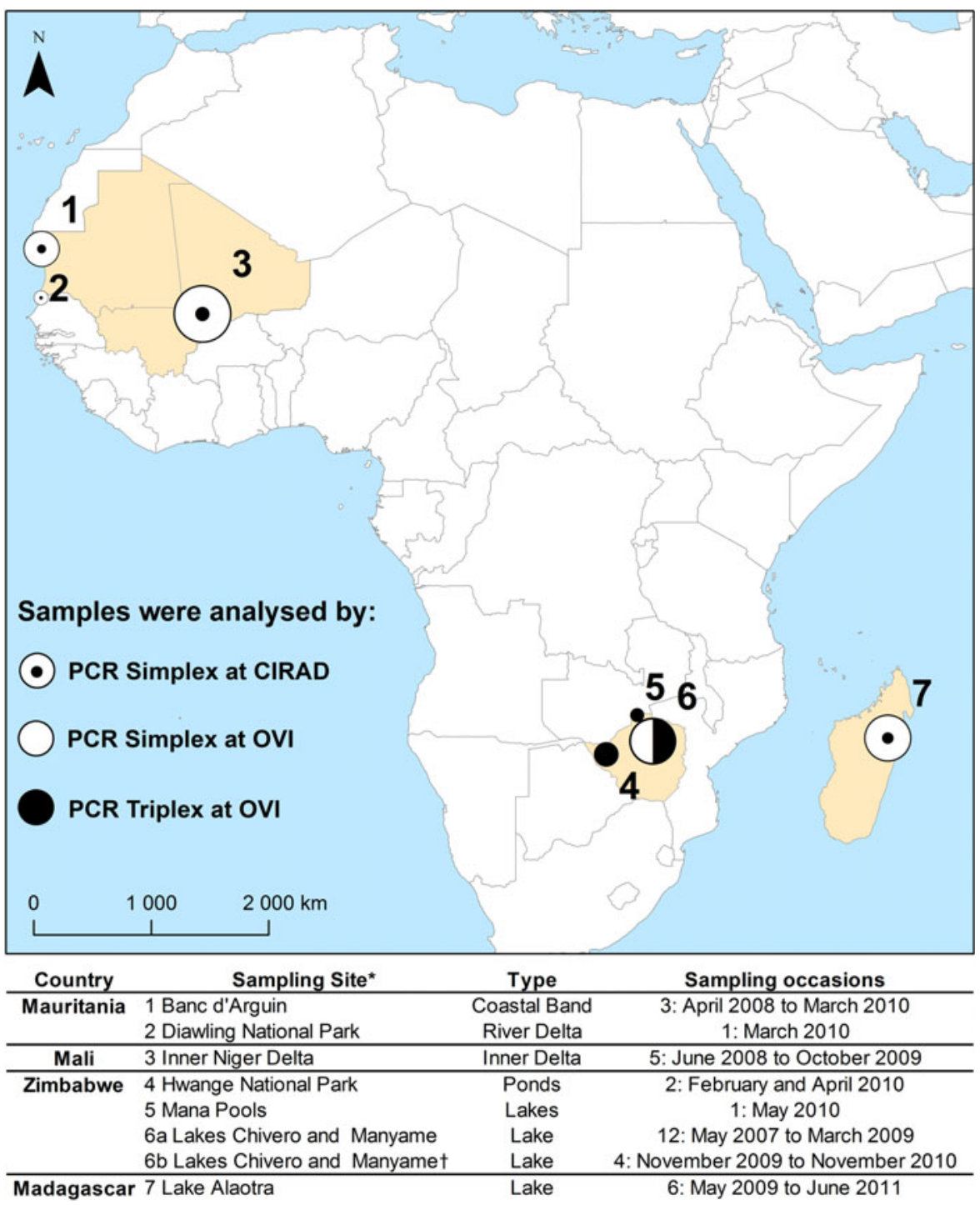

Fig. 1. Map of sample sites showing the seven sites where the 34 sampling occasions of the study took place. It also shows that the samples were analysed in two different laboratories following three different procedures. ${ }^{*}$ The size of the circles on the map is proportional to sampling size of the study site. $\dagger$ Samples collected at lakes Chivero and Manyame were analysed by two different techniques at Onderstepoort Veterinary Institute Laboratory: PCR Simplex for samples collected from May 2007 to March 2009; PCR Triplex for samples collected from November 2009 to November 2010.

were tested by the rRT-PCR method described by Wise et al. [32] whereas samples collected between November 2009 and May 2010 were tested using the method described by Fuller et al. [33]. All rRT-PCRs were run on an Applied Biosystem's StepOnePlus platform (Life Technologies, USA).

\section{Data analysis}

In order to answer the questions (i) and (ii), results from the RT-PCR analyses were summarized to estimate infection rates and their $95 \%$ confidence intervals for each sampling occasion. In order to have an estimation of prevalence with ecological and epidemiological relevance (i.e. with good accuracy and ecological consistency), we merged the results from different wild birds species. We considered six different groups: anatids (Anatidae family: ducks and geese), waders (including Scolopacidae, Charadriidae and Jacanidae families: plovers, sandpipers, snipes, jacanas), gulls (Laridae family: gulls, terns), rails (Rallidae family, e.g. Common moorhen - Gallinula chloropus), Ciconiiformes (including Ardeidae, Pelecanidae and Phalacrocoracidae families: herons, pelican, cormorant), and passerines (including Passeridae and Motacillidae families, e.g. Red-billed quelea-Quelea quelea). 
Studies of AIV infection in wild birds have demonstrated the complementary nature of cloacal and oropharyngeal samples, since birds are rarely found concurrently infected in both types of samples [34]. In our study, all birds were tested for NDV infection in cloacal or faecal sample but not always for tracheal samples. We first compared the results of NDV detection from tracheal $v$ s. cloacal samples in all birds for which both types of samples were available. We then considered only cloacal and faecal samples to conduct our comparative analysis of NDV prevalence between species, seasons and sites in order to account for variation in sampling design. In Zimbabwe in 2007-2008, because of low sample sizes, it was decided to group the sampling occasions into rainy and dry seasons only, leading to four sampling occasions between May 2007 and March 2009. We ended with 24 sampling occasions for the eco-epidemiological analyses.

We tested the effect of two variables on NDV prevalence in a generalized linear mixed model (GLMM). The first explanatory variable with a fixed effect was the type of bird group, a qualitative variable with six modalities (anatids, waders, gulls, rails, Ciconiiformes, passerines). The second explanatory variable with a fixed effect was the season, a qualitative variable with two modalities (dry and rainy). In order to account for a potential country effect, for potential aggregations of infected birds and for a potential difference in diagnostic sensitivity of the three different methods used by the two laboratories of the study, we respectively included 'country' (four modalities), 'sampling occasion' (24 modalities) and 'LabMethod' (three modalities) as variables with a random effect in the model. We also ran four countryspecific models in order to test the effect of the two explanatory variables within each country, with relevant random effect variables (Supplementary Table 1). All models were run using the 'glmer' function in the 'lme4' package of R software [35].

In order to answer question (iii) of our study, we performed phylogenetic reconstruction on short sequences of the F gene (positions 268-443) based on 67 sequences generated from wild bird samples collected in this study and 100 additional sequences retrieved from GenBank. Multiple sequence alignments were performed using the CLUSTAL $\mathrm{W}$ program of the MEGA5 software suite [36]. A sequence from a class I NDV isolate was included as an outgroup to root the tree. The reconstruction was achieved with the Bayesian inference method with a GTR model using MrBayes_3·2·2 [37]. To discriminate between
LPNDV and VNDV, we used the criterion provided by OIE about the sequence of the cleavage site of the fusion protein, according to OIE guidelines, this criterion is sufficient to report an outbreak of Newcastle disease [38]. Therefore, sequences from our study for which multiple basic amino acids have been demonstrated in the virus at the cleavage site of the fusion protein were classified as vNDV.

\section{RESULTS}

A total of 13827 cloacal, tracheal and faecal samples collected from 9085 birds were analysed in this study (Table 1). Overall, $3 \cdot 06 \%$ of the birds were PCR-positive for NDV infection, with prevalence ranging from $0 \%$ to $10 \%$ depending on the season, the site and the species considered (Fig. 2, Supplementary Fig. 1, Supplementary Tables 2 and 3).

Among the 4641 birds for which both cloacal and tracheal samples had been tested, most birds $(n=174)$ were positive for one type of sample only and only a few birds $(n=18)$ were found positive concurrently for both types of samples (Fig. 3, Supplementary Table 4). Furthermore, no significant difference was observed between the proportions of positive cloacal and tracheal samples for the 4641 double-tested birds (Fisher's test, $P=0 \cdot 27$ ).

Regarding question (i), our results showed that a wide diversity of African wild birds were susceptible to NDV infection, with 45 different species testing positive during our study (Supplementary material). Furthermore, 28/29 species with a sample size $>100$ tested positive for NDV infection. The sample size of 100 would give a $95 \%$ probability of detecting a virus circulating at $3 \%$ prevalence (the global NDV infection rate observed in our study) at a given time and site. Among these 29 species, the only species with no NDV-positive sample had the lowest sample size with 108 samples tested. Figure 2 shows that in Mali and Mauritania, where we tested up to six different wild bird species groups, NDV circulates (for given periods and sites) at a similar infection rate in the various groups (Supplementary Fig. 1 shows the same results for all countries). Results from the GLMM did not show significant differences between the prevalence rates measured for the different groups of species considered in our study (Supplementary Table 1).

Regarding question (ii), NDV was detected yearround in wild birds at all the study sites investigated, and we did not detect any NDV-positive sample in only two periods: September-October 2008 in 


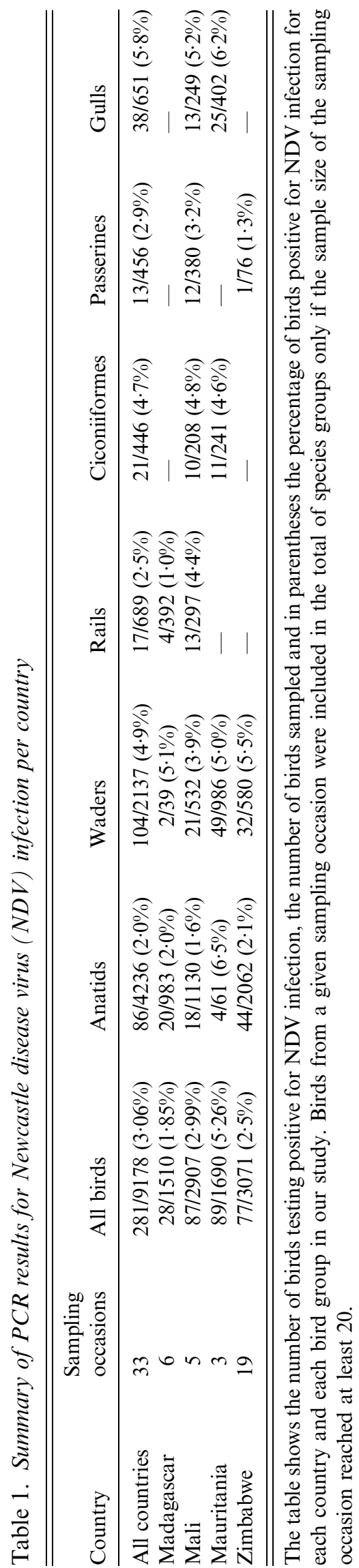

Mali and November 2007-March 2008 in Zimbabwe (Fig. 4). The lack of NDV positives in the Zimbabwe 2007/2008 samples may be attributed to the inability of the Wise et al. assay [32], targetingthe matrix (M) gene to detect class I NDVs. By contrast, the Fuller et al. assay [33] targets the polymerase (L) gene and furthermore applies a dual probe approach to improve sensitivity and specificity. The Fuller et al. assay was validated to detect both class I and II NDVs and was used for the 2008/2009 samples. Our results showed differences in prevalence rates measured for different periods (Fig. 4 and Supplementary Tables 2 and 3). In Mali, results from the GLMM showed that prevalence rates were significantly (GLMM, $P=0 \cdot 005$ ) higher during the dry season than during the rainy season (Supplementary Table 1). This seasonal pattern in Mali is illustrated in Figure 2 which shows decreasing NDV prevalence from dry to rainy season in both 2008 and 2009. In Madagascar and Zimbabwe, there was no significant difference in NDV infection rates between the dry and the rainy seasons (Supplementary Table 1).

Regarding question (iii), from 209 PCR-positive samples from Madagascar, Mali and Mauritania tested for virus sequencing and isolation at CIRAD, three NDV strains were isolated from wild birds while 96 could be partially sequenced for the F gene. However, only 67 sequences were long enough to be included into phylogenetic reconstructions. Results from the phylogenetic analysis of 167 partial sequences of the $F$ gene (176 bp) show that both LPNDV and vNDV are circulating in apparently healthy wild birds (Fig. 5). These strains belong to several genotypes, including African genotypes XI and XIV recently described [4]. Furthermore, closely related strains are circulating in different sites and different wild bird species and are related to LPNDV and vNDV strains circulating in domestic poultry (Fig. 5). We detected vNDV from 17 different species belonging to our six different wild bird groups (Supplementary Table 5). Interestingly, isolates of wild birds are clearly distinct form isolates of poultry in Madagascar (Fig. 5). However, this difference was due to a unique synonymous nucleotide mutation (data not shown).

\section{DISCUSSION}

Our study provides new information on the general eco-epidemiology of Newcastle disease in Africa. First (i), NDV has a large host range and is circulating homogeneously in all groups of wild birds species 

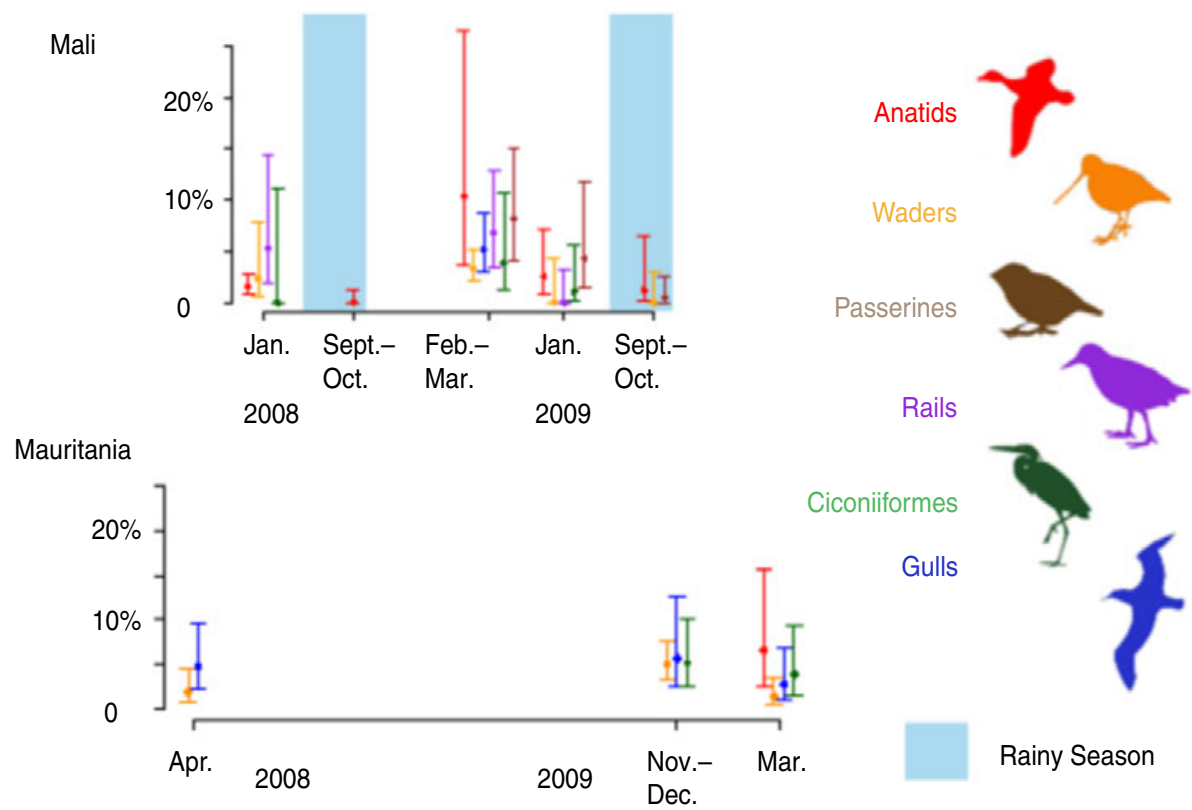

Fig. 2. Homogeneous circulation of Newcastle disease virus in wild birds. The figure shows, for a given sampling occasion, similar prevalence rates with $95 \%$ confidence intervals across all groups of wild bird species based on cloacal and faecal samples in Mali and Mauritania.

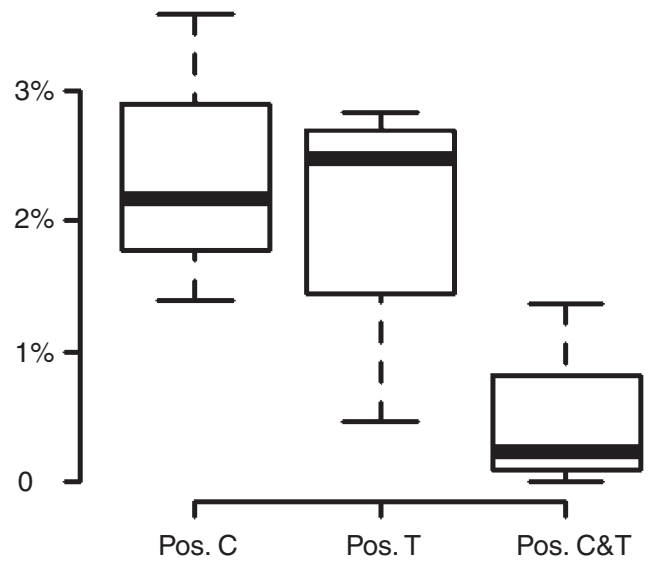

Fig. 3. Comparison between cloacal-faecal and tracheal PCR-positive rates. Boxplot of the PCR-positive rates of samples collected per country on birds that were tested for both cloacal (C) and tracheal (T) samples. A few birds were positive for both cloacal and tracheal samples (C\&T).

tested in our study for a given site and period, with prevalence ranging from $0 \%$ to $10 \%$ across sites and periods of sampling, suggesting potential maintenance of NDV in wild birds. Second (ii), NDV seems to be circulating year-round in wild birds but no consistent seasonal pattern of NDV circulation could be evidenced across all sites, although in Mali, the NDV infection rate was significantly higher in the dry season than in the rainy season during two consecutive years. Last (iii), the phylogenetic analyses of NDV strains isolated in apparently healthy wild birds showed that different genotypes of LPNDV and vNDV circulate concurrently in different species of wild birds at different sites and are phylogenetically related to strains circulating in local domestic poultry. These results suggest that wild birds may play several roles in the epidemiology of different NDV strains in Africa, including vNDV strains potentially responsible for outbreaks in poultry.

Wild birds could participate in the maintenance of NDV in different African regions, including vNDV strains potentially triggering outbreaks in poultry. The large diversity of wild bird species that tested positive for NDV at relatively high prevalence rates suggests the existence of a large maintenance community for NDV as was suggested elsewhere for LPNDV and waterfowl [3]. Our results also suggest a potential large maintenance community for vNDV as 17 different species representing all of our six bird groups tested positive for vNDV infection. As the PCR technique used in Mali, Mauritania and Madagascar targeted class II NDV only, we may have missed class I NDV-positive species and our NDV prevalences may underestimate the actual level of NDV circulation in these countries. In 2009/2010 in Zimbabwe, prevalence rates measured for anatid species ranged from 1.8\% to $5 \%$. In Mali, Madagascar and during the first 


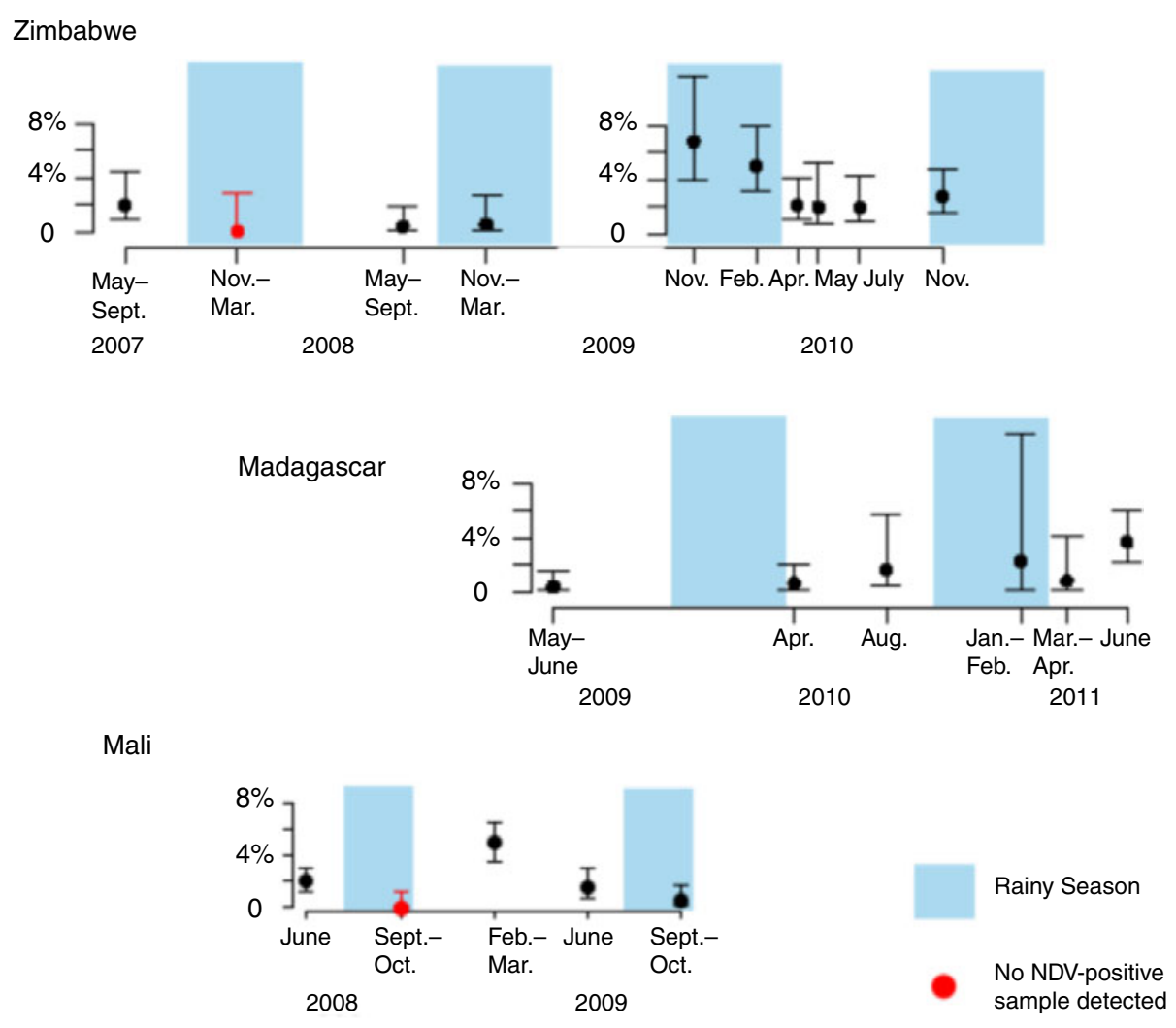

Mauritania

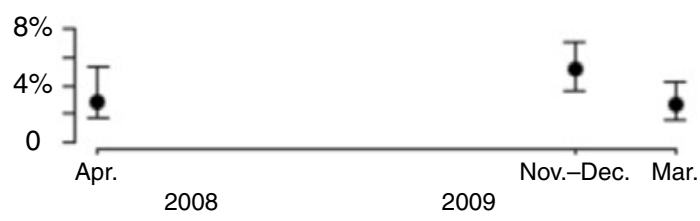

Fig. 4. Seasonal circulation of Newcastle disease virus (NDV) in wild birds. The prevalence rates with $95 \%$ confidence intervals are shown for the wild bird community for each sampling occasion per country based on cloacal and faecal samples. The two periods when no PCR-positive samples were detected are displayed as an open red circle. For Zimbabwe, two different graphs are displayed to reflect the two different PCR methods that were used to analyse the samples.

Zimbabwe study (during which samples were analysed with a different PCR technique) low NDV prevalence rates were recorded at certain times of the year, but positive samples were found all year-round in all countries suggesting persistence of NDV in the wild bird community. Finally, in Banc D’Arguin, Mauritania, a coastal national park with no poultry populations (except in a very few isolated villages) and where high salinity, wind, solar radiation exposure and tidal washing may reduce virus persistence in the environment, prevalence rates measured for all groups of wild bird species ranged from $1.4 \%$ to $6 \cdot 6 \%$ during the study, suggesting continuous inter-individual transmission among wild birds [39]. The difference between PCR techniques used to test the samples prevented us from running a comparative analysis across all sites.
Although vaccinated poultry are considered the main reservoir of vNDV [5-8], the low vaccination rate of backyard poultry in Africa combined with the high mortality and annual loss due to NDV observed in certain African countries [2] may prevent domestic populations from efficiently maintaining the disease. In addition to local trade and wild bird movements, the maintenance and circulation of NDV could then be facilitated by a maintenance community composed of poultry and wild bird populations connected through wild/domestic contacts in the vicinity of African villages [31]. This scenario does not seem to be valid for Madagascar since isolates of wild and domestic birds cluster in distinct branches. However, this difference is supported by a unique nonsynonymous nucleic acid mutation. Therefore, the 


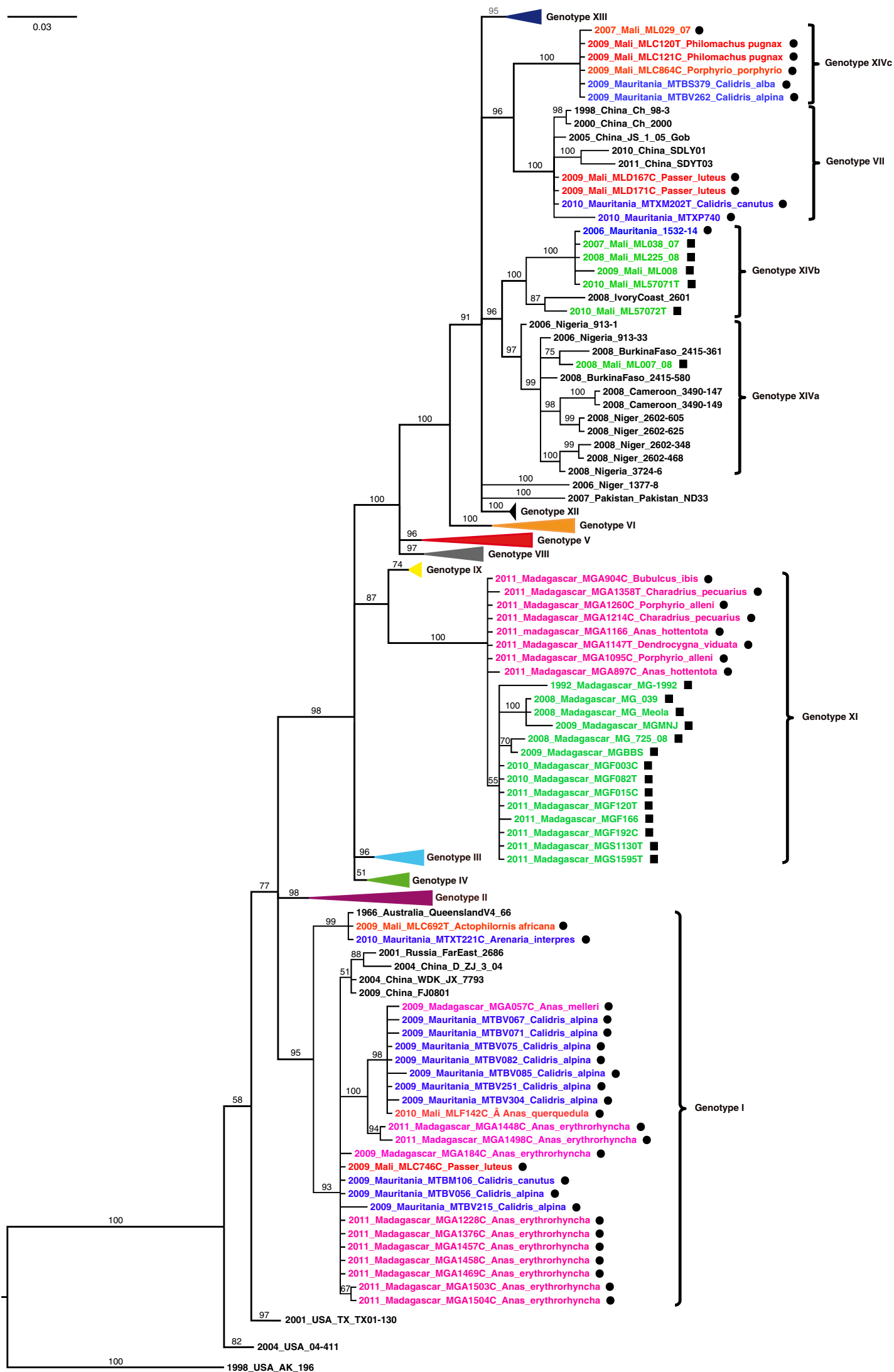

Fig. 5. For legend see next page. 
hypothesis of the existence of a parallel evolution of the same virus between wild and domestic birds, based on this unique acquired mutation, remains speculative. Such a scenario should be at least supported by a higher number of isolates from the two populations collected over a longer period and ideally confirmed by other differences on longer sequences. Furthermore, in the case of frequent transmission of vNDV between domestic and wild birds, direction of viral flow would be difficult to determine and the role of wild birds as a potential reservoir of vNDV would remain speculative.

Wild birds may also participate in the local and regional spread of NDV, connecting different bird populations, including allopatric domestic populations. Closely related strains of vNDV belonging to genotype XIV were identified in Mali and Mauritania, in different wild bird species and in poultry. Among the 17 species in which we detected vNDV, some are known to perform long-distance movement regionally or intercontinentally such as the Hottentot teal (Anas hottentota), the Dunlin (Calidris alpina), or the Slender-billed gull (Chroicocephalus genei) [40]. These results suggest that wild birds may play a role in the local and regional circulation of vNDV strains in West Africa, consistently with the potential role played by Double-crested cormorants in North America [11]. Furthermore, closely related strains of LPNDV belonging to genotype I were identified in Mali, Mauritania and Madagascar, supporting the continental spread of LPNDV by wild birds as suggested previously based on strains detected in Spur-winged geese (Plectropterus gambensis) in Nigeria [41].

Although no consistent seasonal pattern was observed across all sites, the seasonal pattern observed in Mali is consistent with observed seasonality of NDV in backyard African poultry, with epidemic peaks occurring mostly during dry seasons [1]. Both for wild birds in Mali (the present study) and in African backyard chicken [1], NDV circulation is significantly lower during the rainy season. Other studies also showed seasonal patterns of NDV in wild birds. A seasonal peak of NDV was observed in Wood duck in North Carolina, USA during summer when a high proportion of juveniles was present in the population [42]. In North Queensland, Australia, a longitudinal study showed that younger individuals of one duck species were being significantly associated with higher prevalence of NDV, leading to a seasonal peak when these younger birds were commonly sampled [24].

The seasonal pattern observed in Mali is in agreement with the lower circulation of AIV during the rainy season observed in wild birds in Mali [25]. This lower circulation of both AIV and NDV in Mali during the rainy season may be due to strong ecological constraints associated with seasonality. For example, the dispersal of Afro-tropical anatid species during the rainy season for reproduction will likely decrease the contact rates between individuals. On the contrary, during the dry season, the concentration of waterbird species on remnant water bodies, that are also used by other wild and domestic birds, may lead to higher contact and transmission rates [43].

The transmission of NDV is facilitated by the relative stability of the virus in the environment. The virus, including vNDV strains, can survive for long periods in the environment at low temperatures, and for several months in feathers even at relatively high temperatures $\left(20-30^{\circ} \mathrm{C}\right)$ that may be observed in Africa [44]. It is therefore likely that environmental transmission plays a role in the circulation of the virus. Such transmission pattern would be consistent with higher transmission rates between wild and domestic birds when they share remnant water bodies during the dry season. The homogenous circulation of NDV may also be explained by faecal contamination of water sources that are shared by all bird species, including terrestrial species.

Our results demonstrate that collecting and analysing both cloacal and tracheal samples increased the detection rate of NDV in wild birds. This is consistent

\footnotetext{
Fig. 5. Phylogenetic analysis on 167 partial sequence of the $F$ gene nucleic acid sequences of NDV (176 bases). Wild bird sequences from the study are displayed as a black circle and the name of the strain (including the country name) appears in red, blue and purple for strains detected in Mali, Mauritania and Madagascar, respectively. Domestic poultry sequences from the study are displayed as a black square. Trees were constructed using Bayesian inference with 40000000 iterations and 1/1000 trees sampled in the Monte Carlo Markov Chain. A burn-in phase for the first 25\% of tree samples was used to generate the consensus tree. Convergence of the Markov chains was finally checked by an effective sample size (ESS) for all parameters $>6825$ and a potential scale reduction factor (PSRF) within 0.99993 and 1.00007. A class I virus sequence was introduced as an outgroup to root the tree. The final tree was drawn using Figtree 1.4.0 (http://tree.bio.ed. ac.uk/software/figtree/) and the tree posterior probabilities are reported on the branches.
} 
with what is observed for AIV in wild birds and may be explained by the stage of infection or by different preferential sites of infection for different wild birds species and strains, such as HPAIV H5N1 apparent oropharynx affinity [34]. This should be taken into account when designing surveillance programmes of NDV in wild and domestic birds to increase the detection sensitivity.

Conversely to AIV, for which certain wild species like anatids play a major role in the epidemiology of the virus and may then be targeted for surveillance, our study showed that most wild species may play a role in the spread of NDV and no specific group should be targeted a priori. Potential maintenance in the wild bird community is likely vary across regions because of the unique species assemblage, environmental features and cultural practices of each site. Local studies are needed to identify potential transmission routes between wild and domestic species and to implement measures to limit contact and transmission between the wild and domestic compartments [45].

To our knowledge, this study is the first large-scale study on NDV in wild birds in Africa. Our results suggest that all groups of wild bird species may participate in the maintenance of vNDV strains and may potentially be involved in the spread of these strains responsible of outbreaks in poultry. We recommend that any strategic plan aiming at controlling NDV in Africa should take into account the potential role of the local wild bird community in the transmission of the disease.

\section{SUPPLEMENTARY MATERIAL}

For supplementary material accompanying this paper visit http://dx.doi.org/10.1017/S095026881400185X.

\section{ACKNOWLEDGEMENTS}

We thank all our colleagues that participated in the data collection in the field in Madagascar, Mali, Mauritania and Zimbabwe and that analysed the samples at CIRAD and OVI laboratories.

This study, and J. Cappelle, were supported by the GRIPAVI project sponsored by grants from the French Ministry of Foreign Affairs. Data collection in Zimbabwe was partly supported by a USAIDfunded GAINS (Global Avian Influenza network for Surveillance) sub-contract from the Wildlife Conservation Society to G.S.C.

\section{DECLARATION OF INTEREST}

None.

\section{REFERENCES}

1. Miguel E, et al. A meta-analysis of observational epidemiological studies of Newcastle disease in African agro-systems, 1980-2009. Epidemiology and Infection 2013; 141: 1117-1133.

2. Alexander DJ, Aldous EW, Fuller CM. The long view: a selective review of 40 years of Newcastle disease research. Avian Pathology 2012; 41: 329-335.

3. Miller PJ, Decanini EL, Afonso CL. Newcastle disease: Evolution of genotypes and the related diagnostic challenges. Infection Genetics and Evolution 2010; 10: $26-35$.

4. de Almeida RS, et al. New avian paramyxoviruses type I strains identified in Africa provide new outcomes for phylogeny reconstruction and genotype classification. PLOS ONE 2013; 8: e76413.

5. Piacenti AM, et al. Pathogenesis of Newcastle disease in commercial and specific pathogen-free turkeys experimentally infected with isolates of different virulence. Veterinary Pathology Online 2006; 43: 168-178.

6. Miller PJ, et al. Comparison of viral shedding following vaccination with inactivated and live Newcastle disease vaccines formulated with wild-type and recombinant viruses. Avian Diseases 2009; 53: 39-49.

7. Alexander DJ. Newcastle disease in the European Union 2000 to 2009. Avian Pathology 2011; 40: 547-558.

8. Kapczynski DR, King DJ. Protection of chickens against overt clinical disease and determination of viral shedding following vaccination with commercially available Newcastle disease virus vaccines upon challenge with highly virulent virus from the California 2002 exotic Newcastle disease outbreak. Vaccine 2005; 23: 3424 3433.

9. Kim LM, et al. Phylogenetic diversity among lowvirulence Newcastle disease viruses from waterfowl and shorebirds and comparison of genotype distributions to those of poultry-origin isolates. Journal of Virology 2007; 81: 12641-12653.

10. Kuiken T, et al. Excretion of pathogenic Newcastle disease virus by double-crested cormorants (Phalacrocorax auritus) in absence of mortality or clinical signs of disease. Avian Pathology 1998; 27: 541-546.

11. Kuiken T. Review of Newcastle disease in Cormorants. Waterbirds 1999; 22: 333-347.

12. Ujvari D, et al. Phylogenetic analysis reveals extensive evolution of avian paramyxovirus type 1 strains of pigeons (Columba livia) and suggests multiple species transmission. Virus Research 2003; 96: 63-73.

13. Schuler KL, et al. Expansion of an exotic species and concomitant disease outbreaks: pigeon paramyxovirus in free-ranging eurasian collared doves. EcoHealth 2012; 9: 163-170.

14. Kaleta E, Baldauf C. Newcastle disease in free-living and pet birds. In: Alexander DJ, ed. Newcastle 
Disease. Boston: Kluwer Academic Publishers, 1988, pp. 197-246.

15. Kim B-Y, et al. Exchange of Newcastle disease viruses in Korea: the relatedness of isolates between wild birds, live bird markets, poultry farms and neighboring countries. Infection Genetics and Evolution 2012; 12: 478-482.

16. Alexander DJ, et al. Characterization of an antigenically unusual virus responsible for 2 outbreaks of Newcastle-disease in the republic of Ireland in 1990. Veterinary Record 1992; 130: 65-68.

17. Gould AR, et al. Virulent Newcastle disease in Australia: molecular epidemiological analysis of viruses isolated prior to and during the outbreaks of 1998-2000. Virus Research 2001; 77: 51-60.

18. Lindh E, et al. Molecular epidemiology of outbreakassociated and wild-waterfowl-derived Newcastle disease virus strains in finland, including a novel class I genotype. Journal of Clinical Microbiology 2012; 50: 3664-3673.

19. Boynukara B, et al. Determination of Newcastle disease virus among wild bird populations in Lake Van basin, Turkey. Turkish Journal of Veterinary and Animal Sciences 2013; 37: 86-93.

20. Thomazelli LM, et al. Molecular surveillance of the Newcastle disease virus in domestic and wild birds on the North Eastern coast and Amazon biome of Brazil. Brazilian Journal of Poultry Science 2012; 14: 1-7.

21. Snoeck CJ, et al. Characterization of Newcastle disease viruses in Wild and domestic birds in Luxembourg from 2006 to 2008. Applied and Environmental Microbiology 2013; 79: 639-645.

22. Diel DG, et al. Characterization of Newcastle disease viruses isolated from cormorant and gull species in the United States in 2010. Avian Diseases 2012; 56: 128133.

23. Jahangir A, et al. Avian influenza and Newcastle disease viruses from northern pintail in Japan: isolation, characterization and inter-annual comparisons during 20062008. Virus Research 2009; 143: 44-52.

24. Hoque MA, et al. Monitoring of wild birds for Newcastle disease virus in north Queensland, Australia. Preventive Veterinary Medicine 2012; 103: 49-62.

25. Cappelle $\mathbf{J}$, et al. Circulation of avian influenza viruses in wild birds in Inner Niger Delta, Mali. Influenza and Other Respiratory Viruses 2012; 6: 240-244.

26. Caron A, et al. Persistence of low pathogenic avian influenza virus in waterfowl in a Southern African ecosystem. EcoHealth 2011; 8: 109-115.

27. Gaidet $\mathbf{N}$, et al. Understanding the ecological drivers of avian influenza virus infection in wildfowl: a continental-scale study across Africa. Proceedings of the Royal Society, Series B: Biological Sciences 2012; 279: 11311141.

28. Andriamanivo HR, et al. Risk factors for avian influenza and Newcastle disease in smallholder farming systems, Madagascar highlands. Preventive Veterinary Medicine 2012; 104: 114-124.

29. Maminiaina OF, et al. Newcastle disease virus in Madagascar: Identification of an original genotype possibly deriving from a died out ancestor of genotype IV. PLOS ONE 2010; 5.

30. Molia S, et al. Avian influenza and Newcastle disease in three risk areas for $\mathrm{H} 5 \mathrm{~N} 1$ highly pathogenic avian influenza in Mali, 2007-2008. Avian Diseases 2011; 55: 650-658.

31. Cappelle $\mathbf{J}$, et al. Characterizing the interface between wild ducks and poultry to evaluate the potential of transmission of avian pathogens. International Journal of Health Geographics 2011; 10: 60.

32. Wise MG, et al. Development of a real-time reversetranscription PCR for detection of Newcastle disease virus RNA in clinical samples. Journal of Clinical Microbiology 2004; 42: 329-338.

33. Fuller C, et al. Development of an $\mathrm{L}$ gene real-time reverse-transcription PCR assay for the detection of avian paramyxovirus type 1 RNA in clinical samples. Archives of Virology 2010; 155: 817-823.

34. Hoye BJ, et al. Surveillance of wild birds for avian influenza virus. Emerging Infectious Diseases 2010; 16: $1827-1834$.

35. R Development Core Team. R: a language and environment for statistical computing, v. 2.9.0. Vienna, Austria: R Foundation for Statistical Computing, 2009.

36. Tamura K, et al. MEGA5: molecular evolutionary genetics analysis using maximum likelihood, evolutionary distance, and maximum parsimony methods. Molecular Biology and Evolution 2011; 28: 2731-2739.

37. Ronquist F, et al. MrBayes 3·2: Efficient bayesian phylogenetic inference and model choice across a large model space. Systematic Biology 2012; 61: 539-542.

38. World Organization for Animal Health (OIE). Terrestrial Animal Health Code. Newcastle disease, 2012.

39. Gaidet $\mathbf{N}$, et al. Investigating avian influenza infection hotspots in old-world shorebirds. PLOS ONE 2012; 7.

40. Brown LH, Urban EK, Newman K (eds). The Birds of Africa. London: Academic Press, 1982.

41. Snoeck CJ, et al. Genetic diversity of Newcastle disease virus in wild birds and pigeons in West Africa. Applied and Environmental Microbiology 2013; 79: 7867-7874.

42. Goekjian VH, et al. Avian influenza viruses and avian paramyxoviruses in wintering and breeding waterfowl populations in North Carolina, USA. Journal of Wildlife Diseases 2011; 47: 240-245.

43. Cappelle J, et al. Ecological modeling of the spatial distribution of wild waterbirds to identify the main areas where avian influenza viruses are circulating in the inner Niger delta, Mali. EcoHealth 2010; 7: 283.

44. Leighton FA, Heckert RA. Newcastle disease and related avian paramyxoviruses. In: Thomas NJ, Hunter DB, Atkinson CT, eds. Infectious Diseases of Wild Birds. Ames, Iowa, USA: Blackwell publishing, 2007, pp. 3-16.

45. Caron A, et al. Linking avian communities and avian influenza ecology in southern Africa using epidemiological functional groups. Veterinary Research 2012; 43: $1-11$. 